Cinémas

Revue d'études cinématographiques

Journal of Film Studies

\title{
Esthétique du cinéma et relations de cause à effet
}

\section{Laurent Jullier}

Volume 15, numéro 2-3, printemps 2005

Cinélekta 5

URI : https://id.erudit.org/iderudit/012319ar

Aller au sommaire du numéro

Éditeur(s)

Cinémas

ISSN

1181-6945 (imprimé)

1705-6500 (numérique)

Découvrir la revue

Citer cet article

Jullier, L. (2005). Esthétique du cinéma et relations de cause à effet. Cinémas, 15(2-3), 45-61.

\section{Résumé de l'article}

Il est d'usage, dans la critique moderniste de l'art, de dénigrer les relations de cause à effet reliant les deux bouts de la chaîne esthétique, soit la conception et la réception de l'oeuvre. Mais pourquoi le cinéma du shot/reaction shot et de la " théorie des dominos " serait-il mauvais par essence ? Pourquoi devrait-on se sentir honteux, depuis Adorno, de valoriser un film parce qu'il a causé du plaisir ou que sa "valeur d'usage " comprend une certaine utilité ? Il s'agit ici de lutter contre ce type de préjugés, en montrant notamment qu'ils sont issus d'idées non universelles, produites au sein de champs sociohistoriques particuliers, et valorisant une certaine forme d'irrationalisme. 


\title{
Esthétique du cinéma et relations de cause à effet
}

\section{Laurent Jullier}

\section{RÉSUMÉ}

Il est d'usage, dans la critique moderniste de l'art, de dénigrer les relations de cause à effet reliant les deux bouts de la chaîne esthétique, soit la conception et la réception de l'œuvre. Mais pourquoi le cinéma du shot/reaction shot et de la "théorie des dominos" seraitil mauvais par essence? Pourquoi devrait-on se sentir honteux, depuis Adorno, de valoriser un film parce qu'il a causé du plaisir ou que sa "valeur d'usage" comprend une certaine utilité? Il s'agit ici de lutter contre ce type de préjugés, en montrant notamment qu'ils sont issus d'idées non universelles, produites au sein de champs sociohistoriques particuliers, et valorisant une certaine forme d'irrationalisme.

\begin{abstract}
It is common, in modernist art criticism, to denigrate cause and effect relations between the two ends of the aesthetic chain, the conception and the reception of the work. But why must we consider the cinema of shot-reverse shot and the "domino theory" to be essentially bad? Why, since Adorno, should we feel ashamed for appreciating a film because it gives us pleasure, or because its "use-value" consists of a particular utility? As such, the following seeks to combat these prejudices by demonstrating, notably, that they stem from ideas that are not universal, but produced in specific sociohistoric fields, and which uphold a certain form of irrationalism.
\end{abstract}


Parmi les nombreux différends qui séparent les amoureux du cinéma populaire et les exégètes du modernisme cinématographique, les questions relatives à la causalité forment un groupe à part. Le fossé qu'elles ont creusé semble infranchissable, à la fois en ce qui concerne les raisons d'être du cinéma et les raisons d'agir des personnages de films.

D’un côté, le cinéma est supposé procurer de la détente, du plaisir, de l'évasion. Un film doit pouvoir se résumer en quelques phrases et raconter une histoire où, pour toute fumée, existe un feu, et dans laquelle chacun a ce qu'il mérite, la position sociale reflétant le talent, et les actes prêtant toujours à conséquence. Le récit y est construit selon la théorie des dominos, et le shot/reaction shot y fait office de pierre de touche, incitant le spectateur à lire - selon la formule de Roland Barthes - la plus infime consécution en conséquence. Ce mode est économiquement dominant (voir la liste des trois cents plus grands succès de l'histoire du cinéma sur <www.imdb.com >, qui racontent trois cents histoires déterminées par la causalité), et symboliquement dominé (voir les listes des meilleurs films établies par la cinéphilie institutionnelle ou les objets d'étude de l'esthétique du cinéma à l'université). De l'autre côté, le cinéma est supposé dispenser de l'intranquillité. Un film doit mettre le spectateur au défi de dresser la liste des je-ne-sais-quoi irracontables grâce auxquels il a mystérieusement agi sur l'intellect. Il montre des images, fait entendre des sons, les personnages entretiennent avec leur environnement des "liens sensorimoteurs relâchés", comme disait Gilles Deleuze, et l'objet de leur quête - lorsque d'aventure quête il y a — demeure insaisissable. Le shot/reaction shot y est suspect, toujours susceptible de manipuler le spectateur, de lui raconter des histoires, au sens figuré, c'est-à-dire de lui mentir. Ce cinéma de la déconstruction et de l'aléa (figure anti-causaliste que fétichisa Noël Burch dans sa Praxis du cinéma) est économiquement dominé (il ne sort pratiquement des "mondes de l'art» ou du champ expérimental que lorsque des institutions le subventionnent), et symboliquement dominant (tapez par exemple le mot "Godard " dans une recherche sur le Fichier Central des Thèses français, sur < http://fct.u-paris10.fr/ >). 
Loin de désigner d'innocents écarts de goûts et de couleurs, ce différend renvoie donc à des croyances, des opinions politiques ou des façons d'être au monde qui s'opposent radicalement. Mais est-il légitime, vraiment, de faire des relations de cause à effet un tel point de discorde esthétique, éthique et politique? Est-il raisonnable de prêter une visée manipulatrice à leur prolifération dans les films et un pouvoir déniaisant à leur subversion systématique? Le cinéma du shot/reaction shot, qui relève au sens propre de la réaction, en relève-t-il aussi au sens figuré; autrement dit, est-il par essence réactionnaire? Le présent article se propose de donner quelques éléments de réponse à ces questions. Il s'oppose notamment à l'idée de supériorité des histoires déconstruites sur les histoires organisées par la causalité, en essayant de montrer que cette idée repose sur des présupposés biaisés, notamment sur une définition particulariste de l'art.

\section{D’où vient la «mauvaise réputation » idéologique de la causalité?}

Enfants, nous faisons l'apprentissage du monde en cherchant quelles relations de cause à effet l'organisent. À peine un nouveau-né a-t-il ouvert les yeux qu'il tourne la tête en direction de la source d'une voix, puis d'un bruit; à peine un bébé a-t-il gagné assez de coordination musculaire pour saisir un objet qu'il le lâche en vue de vérifier qu'il tombe. Savoir d'où proviennent les sons, prévoir qu'une force pousse inexorablement les choses vers le bas, cela nous permet de nous développer en apprenant à déjouer les pièges de l'environnement. Nous déduisons des lois de ces expériences, sur lesquelles nous nous appuyons pour formuler des hypothèses, faire des prévisions ou inférer tel fait de tel autre, de manière à contrôler le mieux possible ce qui se passe autour de nous. Une fois adultes, nous continuons pour une large part d'interpréter le monde à l'aide de ces lois héritées de la prime enfance, et que la psychologie cognitive regroupe sous le nom de "théories causales naïves" (une forme de knowing that au sens où l'entend Gilbert Ryle, si l'on veut). Dans presque tous nos actes quotidiens nous mobilisons ces universaux; comme le chasseur, nous lisons des traces pour 
reconstruire ce qui s'est passé, comme le détective, nous imaginons des mobiles pour comprendre les actes, quitte à postuler de fantaisistes causes sur de fragiles hypothèses.

Même les plus vastes systèmes de construction de sens mis au point par l'humanité ont pour moteur le désir de remonter à la cause en partant de la conséquence. Les religions et les sciences consacrent ainsi une grande partie de leur programme de compréhension de l'univers à la quête de ses causes premières. Brahmâ, dieu créateur, aussi bien que le Big Bang, consistent à cet égard en des hypothèses causales à très grande échelle de l' "acte d'insufflation" premier. La plupart des mythes apparaissent comme des réponses à l'angoisse devant l'imprévisibilité de l'environnement naturel et de ses manifestations, qu'une théorie causale naïve ne permet pas à elle seule d'expliquer. Toutes les cultures humaines proposent ainsi un équivalent plus ou moins rationnel de Jupiter, c'est-à-dire d'une entité «expliquant» les coups de tonnerre - c'est pourquoi Lévi-Strauss verra le mythe comme une "matrice d'intelligibilité» de l'environnement s'offrant à nous sous différentes formes au fil du temps.

En ce qui concerne l'explication du comportement humain, les candidats ne manquent pas non plus dès qu'il s'agit de trouver des causes. Le modèle conceptuel de la psychologie béhavioriste, le plus connu à ce sujet, reposait, dans ses déclinaisons les plus radicales des années 1950, sur les relations de cause à effet; d'un côté, il y avait l'entrée (input), de l'autre, la sortie (output), et une boîte noire entre les deux, où s'opérait la mécanique causale de réaction de l'être humain à son environnement (on peut encore voir cette théorie exposée par Henri Laborit dans Mon oncle d'Amérique, film de Resnais). Autre exemple: la psychanalyse, qui se propose elle aussi de trouver des causes (inconscientes, cette fois) à ce qui résistait jusqu'alors à l'explication rationnelle (modèle de Freud-Parieto; Freud a d'ailleurs truffé ses écrits de références conceptuelles aux lois les plus causalistes de l'hydraulique et de la thermodynamique). Des disciplines aussi familières que l'informatique et la médecine, encore, sont fondées sur les lois causales: le médecin qui interprète des symptômes remonte des conséquences observables aux 
causes probables (opération connue jadis sous le nom de séméiologie), et l'informaticien écrit ses programmes à l'aide de formules de type "si... alors" (si l'utilisateur entre telle donnée, alors le programme lui renvoie telle autre).

Bref, les relations de cause à effet, théorisées en son temps par Aristote dans sa Métaphysique, imprègnent nos esprits et nos sociétés au point de les constituer en partie. La mauvaise réputation que leurs adversaires leur prêtent vient de l'utilisation politique qui en est faite, notamment lorsqu'il s'agit d'expliquer les différences de condition des êtres humains. Depuis que l'humanité est majoritairement passée des tribus de chasseurscueilleurs aux sociétés agraires, des inégalités de plus en plus en plus grandes ont séparé ses membres. Or pour trouver, comme le dit Max Weber (1996, p. 343), «des réponses rationnellement satisfaisantes à la question de l'origine de la discordance du mérite et de la destinée", différents systèmes de causalité ont d'abord été élaborés. La science, la religion et la philosophie politique en ont chacun produits, tous informés par la causalité: le péché originel, par exemple, a dans le champ de la religion catholique la même fonction explicative que la sédentarisation des tribus de chasseurs-cueilleurs dans le champ de la psychologie évolutionnaire - dans les deux cas, un acte initial mène petit à petit à l'inégalité, à l'envie, au malheur (on trouve en gros la même idée chez Locke et chez Rousseau).

La force physique, qui justifiait la domination chez nos ancêtres les plus lointains, a souvent fait office de système de base: "Qu'un géant et un nain marchent sur la même route, écrivait Rousseau dans son Discours sur l'origine et les fondements de l'inégalité parmi les hommes, chaque pas qu'ils feront l'un et l'autre donnera un nouvel avantage au géant. "On en trouve encore l'écho, parfois, dans l'explication des inégalités qui séparent hommes et femmes au sein des classes sociales où la force de travail est le seul capital à vendre. La trace s'en fait également sentir dans les représentations populaires des superhéros de la bande dessinée et du cinéma, tous plus musclés les uns que les autres... Si cette causalité avait un poids explicatif il y a plusieurs milliers d'années (le chef, comme dans certains groupes d'animaux, était l'individu le plus fort), elle l'a 
évidemment perdu. Nous sommes dirigés par des gens dotés d'une force physique moyenne, sinon négligeable: le gouverneur de Californie, Arnold Schwarzenegger, fait figure d'exception. Encore sa musculature n'a-t-elle pas été causée par une action mécaniquement utile à la communauté, mais par un entraînement adéquat - l'image de la force à défaut de sa fonction, figure typique de l'ère postmoderne.

Bien entendu, la force physique n'est pas le seul élément qu'on retient dans la course à l'explication des inégalités par le sens commun. Les religions, en tous temps et en tous lieux, ont pour ce faire mis au point de complexes systèmes, tantôt stochastiques (où la fatalité, orchestrée d'une main négligente par un dieu joueur de dés, nous fait naître ici ou là, petit ou grand, etc.), tantôt régis par la théorie des dominos (comme le système des réincarnations "méritées" de l'hindouisme). Comme le remarqua Max Weber, non sans ironie, ces récits rendent "un service de légitimation à tous les dominants, les possédants, les vainqueurs, les biens portants, bref tous les heureux ». Car :

$$
\begin{aligned}
& \text { [...] l'homme heureux se contente rarement du fait } \\
& \text { d'être heureux; il éprouve de surcroît le besoin d'y } \\
& \text { avoir droit. Il veut aussi être convaincu qu'il mérite son } \\
& \text { bonheur, et surtout qu'il le mérite par comparaison } \\
& \text { avec d'autres. Et il veut donc également croire qu'en ne } \\
& \text { possédant pas le même bonheur, le moins fortuné n'a } \\
& \text { que ce qu'il mérite (Weber 1996, p. 337-338). }
\end{aligned}
$$

On ne saurait être plus clair sur l'importance de la causalité - voilà, donc, que se dessine une première explication de sa "mauvaise réputation».

L'éternel débat entre l'inné et l'acquis - une sous-classe de la lutte entre l'essentialisme et le déterminisme — a donné la dernière touche à cette réputation. Les essentialistes situent traditionnellement les causes de la supériorité dans le talent de l'individu, tandis que les déterministes les voient plutôt dans le contexte social de sa naissance et de sa vie.

Les premiers professaient jadis le "chacun selon son dû"; l'écho s'en trouve dans le darwinisme social des grands capitaines d'industrie états-uniens (John Carnegie, John D. Rockefeller) aussi bien que dans l'image du clochard bohème 
qui "préfere» ne pas travailler. Ici, le statut social est proportionnel à la volonté (will power) et aux aptitudes "naturelles" (natural leadership). Le puissant, ici, l'est parce qu'il le mérite, parce qu'il a des dons - si ce n'est pas Dieu qui les lui a conférés, c'est qu'il s'est «fait tout seul ». Herbert Spencer, à l'origine du darwinisme social américain, faisait mine d'en appeler au hasard pour justifier les inégalités, mais se fondait sur une causalité proprement génétique: les pauvres gens ont tiré le mauvais numéro dans la grande loterie de l'hérédité biologique... Les sociologues français de la grande bourgeoisie, Michel Pinçon et Monique Pinçon-Charlot (1989), ont d'ailleurs remarqué dès le début de leurs enquêtes combien les parents dans les "grandes familles" dissimulent le travail d'éducation de leurs bambins (ils ne les grondent jamais en public, par exemple), laissant à penser que la chair de leur chair sait "naturellement" maîtriser les codes sophistiqués qui, du polo à la gestion des portefeuilles d'actions en passant par le maniement désinvolte des couverts à poisson, feront l'ordinaire de leur vie future. De nombreuses fictions relaient cette explication innéiste des inégalités (conjuguée à une conception lamarckienne de la transmission génétique); ainsi Superman, Harry Potter et Luke Skywalker, ces champions du cinéma populaire, ont-ils reçu d'un puissant père le don qui leur permettra causalement d'être number one chacun à leur manière.

Les contextualistes, au contraire, pensaient que l'environnement déterminait grandement la probabilité de "réussir sa vie». La morale des Animaux malades de la peste, "selon que vous serez puissant ou misérable...", leur servait d'étendard. Selon que vous naissiez fils de haut fonctionnaire ou fille de maçon, les chances d'occuper une position dominante étaient différentes, à will power égal. Bien entendu, le darwinisme social brandissait toujours quelques histoires à la Carnegie de cireurs de chaussures devenus magnats de la finance, mais ces exceptions (quoiqu'elles soutiennent probablement presque entièrement l'American dream ou le rêve de "s'en sortir" dans un grand nombre de pays) ne valent que ponctuellement, pas statistiquement. Les déterministes considéraient les individus comme le produit du contexte de leur venue au monde et de leur 
éducation, ce qui expliquait le natural leadership, c'est-à-dire, plus prosaïquement, le talent dans la gestion d'un portefeuille d'actions héritées du père. Cette vision s'accorde mieux avec une vision darwinienne, cette fois, de la transmission génétique. Elle recoupe également une autre constatation faite par les Pinçon: un "nouveau riche" (par exemple, le fameux cireur de chaussures changé en magnat) ne pourra intégrer les cercles des puissants que s'il a fait la preuve (et de préférence sur plus d'une ou deux générations) de sa capacité à transmettre sa puissance à ses descendants. Si vraiment cette transmission était directe, lamarckienne et automatique, un tel examen de passage n'aurait pas lieu d'être, peuvent conclure les contextualistes...

Au final, le résultat est pourtant le même, que la transmission se fasse majoritairement par le biais de l'inné (Luke Skywalker) ou de l'acquis (Batman et Spider-Man, encore inexpérimentés, recueillant in extremis de la bouche d'un mourant - Batman de son père, Spider-Man de son oncle - le sage mantra qui, une fois assimilé, leur permettra de donner un but à leur vie, un sens à leur talent). Bref, les explications causales rendent fatalistes. Relayées par le cinéma populaire, elles répondent à un désir de présenter comme ce-qui-va-de-soi une organisation sociale qui profite à la bourgeoisie. On lit alors le «message» de ce cinéma comme un système factuel alors qu'il s'agit d'un système sémiologique, on le vit comme une parole innocente alors qu'il travaille à naturaliser un concept artificiel en le «vidant d'histoire pour le remplir de nature", comme l'écrivait Barthes dans ses Mythologies. Voilà d'où vient la "mauvaise réputation" des Grands Récits de la causalité directe.

\section{Réduction 1 : du monde social à la question de l'effet-clip au cinéma}

Chez Adorno, dans la foulée de Marx et de Weber, la rationalisation de la société a abouti à faire de la vie "un épiphénomène de la production matérielle». La machine à commettre, au sens où l'entend Heidegger, tourne désormais toute seule chez nous, et réalise la "transformation des sujets en fonctions sociales" (Adorno 1991, p. 192) - l'expression "ressources humaines" en est le reflet... Max Weber (1996) l'avait déjà décrite sous le 
nom de "cage de fer", et Charles Taylor (1994) a repris cette idée sous le nom de "primauté de la raison instrumentale».

Or, chez les critiques modernistes, le cinéma du shot/reaction shot et des héros occupés à mener à bien leur quête passe pour refléter cette calamiteuse raison instrumentale — c'est pourquoi Adorno ne voyait guère de différences conceptuelles entre aller au bureau et aller au cinéma. Dans la foulée de Clement Greenberg, utilisant les mêmes idées, les apologues du cinéma de la modernité (Nouvelle Vague, Free Cinema, etc.) ont opposé à ce cinéma celui de la déconstruction, de l'errance, du doute. Plus récemment, renouvelant quelque peu ce fonds de commerce esthétique, une partie des critiques d'inspiration culturaliste s'est mise à défendre des images plus accessibles et moins radicales au nom du même principe, des images qui participent de l'effet-clip - travellings avant à toute vitesse, feux d'artifice de plans $l o-f i$ au cœur d'une bagarre, explosions en THX... Au moins, pendant qu'il regarde de tels feux d'artifice, le spectateur est libre de laisser son esprit vagabonder... Steve Bukatman (1998) parle ainsi de "séquences kaléidoscopiques" qui provoquent une expérience spectatorielle proprement kinésique, et dont le prototype est la séquence du Stargate Corridor dans 2001, l'Odyssée de l'espace. Ces séquences proviendraient en ligne directe d'une longue suite de trouvailles, de jouets et de dispositifs qui permirent en leur temps de lutter contre l'excès de rationalité des Lumières. Selon la formule de Geoffrey O'Brien, que cite Bukatman (1998, p. 82), le cinéma s'attache dans ces morceaux de bravoure audiovisuels à "reconstruire les mondes imaginaires qu'il a contribué à démanteler ". La caméra ne serait donc, après le kaléidoscope, la photographie spirite, les montagnes russes et les dioramas, que le dernier avatar d'un refoulé des Lumières qui n'en finirait plus de remonter... Paradoxalement, dit Bukatman (1998, p. 92-93), la débauche de technique nécessaire pour produire les effets spéciaux kaléidoscopiques permet au spectateur d'avoir une "activité anti-rationnelle et anti-téléologique». Cette "transgression kinésique utopique», proche de la dérive situationniste, lui permettrait alors de se libérer de l'environnement technocratique qui est le sien, autrement dit de sortir par l'imagination de la "cage de fer». 
Les remarques de Bukatman s'appliqueraient mieux aux synthétiseurs d'images qui déploient leurs fastes miroitants dans les rave-parties, car tous les morceaux de bravoure des blockbusters hollywoodiens sont en fait conçus en fonction d'un but, restant de ce fait prisonniers d'une logique narrative sousjacente. Le public des blockbusters apprécie la pause feux d'artifice comme celui des musicals hollywoodiens appréciait jadis la pause danses et chants: parce qu'il ne s'agit que d'une suspension provisoire de l'enchaînement causal des péripéties. Et puis, surtout, les spectateurs sont libres de penser à ce qu'ils veulent quoi qu'il se passe sur l'écran: ce n'est certes pas parce qu'il y a un trésor à trouver ou une princesse à sauver que le spectateur est exclusivement occupé à se demander par quels moyens le héros parviendra à ses fins, de la même façon qu'au bureau (pour revenir à la comparaison chère à Adorno), l'employé ne se demande pas constamment par quels moyens rendre à son chef tel rapport urgent. Le vieux fantasme du spectateur passif, lieu commun vieux d'un siècle, est, on le voit, toujours prêt à resurgir... Mais nous n'avons pas besoin d'un long plan fixe sur un visage pensif ni de distanciation brechtienne tous azimuts pour nous inciter à réfléchir, à nous montrer actifs ou vigilants. D'un super-héros aux prises avec un savant fou, d'un amoureux qui trébuche dans une comédie sentimentale, nous pouvons aussi nous demander, comme Saadi entendant vanter les exploits de Gengis Khan, s'il aime les roses... Les projections (intimes) que nous faisons sur les projections (techniques) du cinéma dépendent de facteurs en nombre bien plus grand que ceux qui concernent les formes narratives...

\section{Réduction 2 : la question du jugement de goût au cinéma}

La suspicion des critiques modernistes à l'égard des pouvoirs explicatifs des mécanismes de causalité les a également fait préférer, comme base du jugement de goût porté sur les films, l'intuition chère à Kant à la rationalité axiologique chère à Max Weber. À la lumière du système élaboré par le gentilhomme de Königsberg, le beau film est celui qui possède des qualités inaccessibles à la logique et au monde des émotions — donc des qualités non causées au sens mécanique. Ces qualités in- 
connaissables, accessibles seulement aux privilégiés qui forment la communauté des gens de goût par le truchement d'une mystérieuse intuition, peuvent même échapper au "chacun ses goûts" relativiste; selon la Critique de la faculté de juger, écrite en 1790, elles transcendent à la fois la science et le plaisir sensuel. On peut vérifier chaque mois que les cinéphiles professionnels refusent la démarche causaliste (ils n'utilisent pas de grille d'évaluation) et se méfient tout autant des émotions (le corps n'est pas un instrument de mesure fiable, bien plutôt la chair est faible, provoquant des réactions automatiques). Au lieu de cela, au lieu d'être analysé (tel plan est raté parce que...) ou commenté de façon non déguisée (j’ai ri, j’ai pleuré comme un veau...), le film est re-dit. Ils en extraient un certain nombre de particularités pour les traduire en mots et les réorganiser de façon à construire un "texte-bis» (comme disaient les déconstructionnistes). Ce texte-bis, résultat de ce que David Bordwell appelle dans son livre éponyme making meaning (fabriquer du sens), s'accorde en fait à l'habitus et au «bon goût» de leur communauté. Ils s'y reconnaissent; en échange, le texte construit leur identité de tribu. On voit la circularité dans cette démarche - Bourdieu a attiré l'attention sur elle des dizaines d'années durant...

Les récents travaux du sociologue Bernard Lahire tendent à faire croire que toutes ces tribus, désormais, ont des contours flous, au point qu'on ne peut étudier que la culture des individus. Sans doute y a-t-il de pauvres gens sans diplôme, travail ni domicile qui adorent le cinéma de Jean-Luc Godard, mais il est tout de même un peu léger de laisser tomber toute idée statistique reposant sur la notion de "plus grande chance" sous prétexte qu'il existe quelques contre-exemples. En ce qui concerne le bagage nécessaire à la compréhension et à l'appréciation d'un film, il y a beaucoup moins de différences entre un homme et une femme (ou un Noir et un Blanc), qu'entre un riche et un pauvre (ou qu'entre un habitant du sixième arrondissement de Paris et un habitant de la banlieue de Bombay), car il faut réunir quatre conditions pour apprécier le genre de films que plébiscite la critique néokantienne: 1) la présence de ces films à proximité du lieu de résidence; 2) le temps libre que requiert leur 
visionnage (car le cinéma impose sa durée au spectateur); 3) l'absence de préjugés culturels et l'ouverture d'esprit qui s'acquièrent grâce à la fréquentation dès l'enfance des objets d'art les plus variés; 4) un environnement affectif et social qui apporte une forme de reconnaissance (non une forme de marginalisation) à qui consomme ce genre de produits. Pour apprécier le dernier Godard à la hauteur où le place habituellement la critique néokantienne, il faut, par exemple: 1) habiter une grande ville; 2) disposer de deux heures et des moyens de s'offrir la place; 3) n'attendre pas d'un film qu'il raconte une histoire sur le modèle classique du récit-quête avec symétrie de clôture et en fonction de la théorie des dominos; 4) lire des articles qui expliquent combien ce film est l'une des créations les plus élevées du génie humain, et avoir des amis qui l'ont vu eux aussi (pour ne pas s'attirer des yeux écarquillés ni des "mais pourquoi diable es-tu allé voir une chose pareille?»).

Or le critique néokantien pense manifestement que tout le monde vit comme lui (ou aimerait en tous cas vivre comme lui). Lorsque Alain (1949, p. 41), dans ses Propos sur l'esthétique, dit qu'il a «connu un prolétaire qui courait au musée du Louvre dès qu'il pouvait dérober une heure» dans l'espoir (jamais réalisé) de «recevoir la grâce» devant les tableaux, il ne fait que la moitié de l'analyse: c'est justement parce que ce malheureux est obligé de dérober une heure - au lieu de baigner à loisir dans le climat d'aficion du grand art pictural — que Rembrandt ne lui dit rien...

La circularité de la situation, à nouveau, frappe par son évidence. En premier lieu, les récits déconstruits sont justement ceux qui ont besoin d'explications, donc de "passeurs", d'exégètes professionnels, d'herméneutes (tandis que les histoires orchestrées par des relations de cause à effet relèvent d'universaux pour untutored audience, comme le dit Noël Carroll). En second lieu, le seul mode d'expertise qui permette au passeur de rester "celui qui sait» est le jugement intuitif kantien (au mieux, par répétition et imprégnation, le connoisseurship d'un passeur peut aboutir à la formation de disciples - tandis que le méthodologue qui utilise les relations de cause à effet pour expliquer la valeur des films, une fois qu'il a fait voir ses outils, n'est plus «celui qui sait»). Pour une grande partie, et sans 
verser dans un déterminisme à tout crin, c'est la place sociale réelle du critique qui apparaît comme l'explication utilitariste la plus rationnelle.

Le plus gros problème reste là aussi l'idée - tant ressassée implicitement article après article qu'elle en devient terreur théorique - d'une supériorité du high art déconstruit, et pouvant être goûté intuitivement, sur le film populaire accessible à tous. Primo, comme on vient de le voir, chercher à séparer les images animées "artistiques" des autres est en réalité un exercice stérile et ennuyeux, à moins de faire partie du monde de l'art et de voir là un moyen de gagner son pain. Secundo, il est toujours possible de se servir de la disposition esthétique pour recevoir un objet qui n'a pas été pensé comme une œuvre d'art. Par exemple, le regard d'André Bazin — qui est resté marginal — lui permettait de trouver tout de même son bonheur en visionnant un navet, dans la simple exactitude biomécanique des images des êtres qui défilaient sur l'écran. Pour provoquer une réception "esthétique ", il faut bien plus souvent un film qui torpille ouvertement les codes narratifs ou représentationnels, sinon, en guise d'encouragement, le contexte d'une salle sélect... Tertio, la définition du high art est ici une définition particulariste, adaptée à un champ et à une époque, et ne relève nullement des universaux (il n'y a donc pas de raison de se sentir "terrorisé » à l'idée qu'on n'a strictement rien compris au dernier Godard).

Un récent courant de pensée anglo-saxon, qui appelle l'éthologie et la biologie évolutionniste au renfort de l'esthétique, se propose de lutter contre cette "terreur». L'une de ses représentantes est Ellen Dissanayake, dont le livre-phare What Is Art For? (littéralement pour quoi l'art existe), s'ouvre sur cette constatation fort simple: les arts sont un phénomène culturel, mais l'Art est une priorité biologique. Dissanayake entend dépasser ce qu'elle appelle la "définition occidentale-avancée» de l'art, qui postule que celui-ci ne doit servir à rien, être fait pour rien sinon pour lui-même, et appeler la consommation désintéressée des gens de goût. Car cette définition ne tient compte que d'une très courte et très récente période de l'histoire de l'art.

Son programme universaliste nous permettra, dans le cadre présent, de combiner plus facilement les notions d'expérience 
esthétique et d'objet artistique pour les adapter sans honte au cinéma - la honte de parler d'art à propos de produits si ostensiblement faits pour plaire, pour être loués ou achetés sous forme de copies DVD... On retiendra dix caractéristiques de l'art parmi celles qu'elle propose, dix «ressemblances de famille» que possèdent séparément certaines caractéristiques du comportement humain, mais qui se rencontrent souvent ensemble dans un grand nombre de formes d'art par-delà les siècles et les continents, et que le cinéma - ainsi qu'un bon nombre d'autres types d'images animées - possède toutes. Ce sont des causes d'amour des œuvres, qui se passent de l'intuition kantienne.

- Le témoignage d'habileté. Le savoir-faire technique est une valeur populaire que la critique cinéphilique regarde aujourd'hui de haut. Mais l'utilisation d'images de synthèse photoréalistes, tout comme la faconde de tel acteur ou l'élégance de geste d'un cadreur, sont des motifs suffisants pour attribuer de la valeur à un film.

- Le plaisir sensuel. Personne ne contestera que le cinéma, et ses cousins postmodernes comme le vidéoclip, fassent effet de ce côté.

- L'expérience sensorielle ressentie comme telle. Parfois, le film nous «dé-familiarise» en nous faisant voir le monde avant son filtrage par l'habitus, cet ensemble de routines perceptives qui nous semblent "aller de soi" alors qu'elles sont construites par notre position sociale. Les formalistes soviétiques théorisaient cette défamiliarisation sous le nom d'ostranienie: l'ouvrier devant lequel Dziga Vertov projetait un reportage était censé n'avoir jamais vu les choses sous cet angle, au propre et au figuré.

- L'harmonisation, la mise en ordre. Donner une unité à ce qui a sur le coup été perçu comme une collection désordonnée d'événements: le montage même de deux plans consécutifs suggère déjà un lien entre le contenu de ces plans. Intolerance, par exemple, le fameux film de D. W. Griffith, était une grandiose tentative d'unification de deux mille ans d'histoire occidentale (même si la plupart de ses spectateurs pensèrent qu'elle avait échoué).

- L'innovation. Produire des images, des sons et des combinaisons audiovisuelles qui n'ont encore jamais été vues ni 
entendues. N'oublions pas que la néophilie moderniste, qui valorise surtout les remises en cause radicales des langages de l'art, n'est pas la seule valable. Les sorciers de l'informatique graphique, qui fabriquent des mondes et des créatures de synthèse, relèvent tout autant de cet élan innovant.

- L'embellissement. Rendre les choses plus belles. Ne filmer un comédien que sous son meilleur profil. Faire repeindre l'herbe (Kaurismaki) ou une palissade (Carax), nettoyer Paris (Jeunet), au risque de mentir et de faire craindre le retour à la réalité... Encore une valeur populaire que la critique cinéphilique charge de bien des maux...

- L'expression. Comme disait Lev Vygotski dans les années 1920, l'art est une technique sociale du sentiment, c'est pourquoi sa fonction expressive a de l'importance. Elle peut mener au culte de la personnalité (la "politique des auteurs" chère aux Cahiers du cinéma), ou, mieux, tenir lieu d'expérience amicale, comme si le spectateur, au sortir du film, avait rencontré une personne désireuse de lui confier quelque chose: une conviction, un secret, un souvenir, une clé parfois pour vivre mieux.

- L'artifice. Par opposition au donné naturel, aux contraintes biomécaniques, aux lois qui font que tout finit par passer, l'art propose un monde alternatif. Les samouraïs font des bonds de vingt mètres, des justiciers masqués extirpent le mal des grandes cités, le mariage est heureux jusqu'au bout: les mondes possibles du cinéma se fondent sur le réel mais consolent souvent de ses limites et de ses avanies. Parfois c'est le contraire: l'artifice accentue la violence des lois dominantes, et la consolation arrive lorsqu'on retourne à la vraie vie au lieu d'être donnée durant la projection.

— Le jeu, le "faire comme si" (make-believe). "On dit que t'es Spider-Man...»Qui mieux que le cinéma réussit à nous refaire jouer à être quelqu'un d'autre? Il suffit de se retourner, depuis le premier rang, pour regarder une salle comble en train de suivre la poursuite sur une autoroute de Matrix Reloaded pour s'assurer que l'enfance n'est jamais moins loin que devant un grand écran. De surcroît, le jeu étant le "travail de l'enfant» — c'est-à-dire la monnaie d'échange de son apprentissage de 
l'environnement —, grâce à ces emprunts momentanés d'identités, nous acquérons parfois quelques savoirs utiles ou obtenons des clarifications bienvenues (le film nous a donné un exemple illustrant une idée qui nous semblait valable mais qu'il nous était impossible de formuler clairement).

- L'élévation existentielle. De la plus modeste - le divertissement - à la plus haute - l'expérience extraordinaire, l'extase, la naissance d'une vocation, la conversion... Les romantiques ont porté cette caractéristique au pinacle, et Stanley Cavell a écrit des centaines de pages pour expliquer que le cinéma avait fait de lui un homme meilleur...

\section{Conclusion : pour un surcroît de prudence heuristique}

Non seulement la logique causale n'est pas par essence condamnée à accompagner, dans la conception et dans la réception artistiques, des sous-produits abêtissants, mais elle vaut mieux que sa réputation ne le laisse entendre.

Certes, l'histoire de la philosophie a été marquée par l'abandon progressif des explications radicalement causalistes du cours des choses (Kistler 2004). Le coup d'envoi d'une conception véritablement désenchantée de la causalité a été donné, il y aura bientôt un siècle, par Bertrand Russell, dans un article à peu près contemporain de la mécanique quantique - théorie qui sous-tend l'idée que Dieu joue bien aux dés, quoi qu'en pensait Einstein ("On the concept of cause", 1912). Le terrain, dès lors, se trouvait préparé pour les théories "prudentes" de la causalité $\mathrm{du} \mathrm{XX}^{\mathrm{e}}$ siècle, comme la théorie de la manipulabilité, celle des probabilités ou la plus prudente de toutes, influencée par les lois de la physique quantique, la théorie du transfert, où la causalité réside dans «la capacité de $\mathrm{A}$ à transférer un marqueur à B, attestant de la continuité d'un processus" (Salmon 1984, p. 147).

Pourtant la causalité est présente, y compris celle des théories causales naïves, au creux des réalisations techniques les plus sophistiquées - la robotique et l'intelligence artificielle ellesmêmes sont empreintes de physique naïve, une discipline contemporaine de la phénoménologie, et tout aussi soucieuses qu'elle de traiter de phénomènes observables à la seule échelle 
humaine et pouvant être appréhendés par le sens commun (Smith et Casati 1993). De plus, l'homme de la rue est comme le philosophe et le scientifique: il utilise des déclinaisons des relations de cause à effet bien plus complexes que le simple si... alors du domino qui pousse en tombant l'autre domino devant lui. La psychologie cognitive a posé de sérieux bémols à la conception béhavioriste-causaliste, et a montré qu'un grand nombre des images mentales qui nous viennent à l'esprit sont gouvernées par des lois probabilistes (Edelman 1992). Par exemple, nous utilisons énormément les tirages probabilistes et les inférences statistiques dans notre vie de tous les jours, bien plus souvent que la déduction rationnelle basée sur la chaîne de cause à effet (Rapport 2000) — inutile de préciser qu’il en va de même devant un film, même un western de l'âge d'or.

\section{Université Paris III}

\section{RÉFÉRENCES BIBLIOGRAPHIQUES}

Adorno 1991: Theodor W. Adorno, Minima moralia. Réflexions sur la vie mutilée [1951], Paris, Payot, 1991.

Alain 1949: Alain, Propos sur l'esthétique, Paris, PUF, 1949.

Bukatman 1998: Steve Bukatman, "The Ultimate Trip: Special effects and Kaleidoscopic Perception ", Iris, n 25, 1998, p. 75-97.

Dissanayake 1988: Ellen Dissanayake, What Is Art For?, Seattle, University of Washington Press, 1988.

Edelman 1992: Gerald Edelman, Bright Air, Brilliant Fire: On the Matter of the Mind, Harmondsworth, Penguin, 1992.

Kistler 2004: Max Kistler, "La causalité dans la philosophie contemporaine", Intellectica, vol. 1, n 38, 2004, p. 139-185.

Pinçon et Pinçon-Charlot 1989: Michel Pinçon et Monique Pinçon-Charlot, Dans les beaux quartiers, Paris, Seuil, 1989.

Rapport 2000 : Nigel Rapport, "Random Mind: Towards an Appreciation of Openness in Individual, Society and Anthropology", Australian Journal of Anthropology, août $2000,<$ http://www.aas.asn.au/TAJA.htm >.

Salmon 1984: Wesley Salmon, Scientific Explanation and the Causal Structure of the World, Princeton, Princeton University Press, 1984.

Smith et Casati 1993: Barry Smith et Roberto Casati, «La physique naïve: un essai d'ontologie», Intellectica, vol. 17, n 2, 1993, p. 173-197.

Taylor 1994: Charles Taylor, Le malaise de la modernité [1991], Paris, Le Cerf, 1994. Weber 1996: Max Weber, Sociologie des religions [1910-1920], Paris, Gallimard, 1996. 